\title{
Infections in Acute Pancreatitis: A Review
}

\author{
Chetana Vaishnavi ${ }^{1}$, Nikhil Bush ${ }^{2}$, Rakesh Kochhar ${ }^{3}$
}

\begin{abstract}
Acute pancreatitis (AP) is an unpredictable disease accounting for more than $50 \%$ of all hospital admissions for pancreatic diseases. The disease is generally mild and self-limiting, but about $20 \%$ of the patients develop moderate to severe AP with poor prognosis leading to high morbidity and mortality. Recently increased occurrence of AP has been noted with severe AP requiring several weeks to months for treatment. Acute pancreatitis may get complicated by pancreatic and/or peripancreatic necrosis, which may become infected through several routes. The pathogenesis of secondary bacterial pancreatic infection is still under controversy. Acute pancreatitis is the setting for most infections of the pancreas, which can complicate various stages of pancreatitis. In some patients, AP develops into necrotizing pancreatitis. Patients with AP-related infected pancreatic necrosis (IPN) are prone to develop organ failure, leading to increased mortality, further complicated by multidrug-resistant (MDR) microorganisms. The bacterial spectrum in IPN reflects the commensal flora of the intestine. Clinically, there are no reliable parameters to distinguish infected from sterile necrosis as the clinical features are indistinguishable from other infectious conditions. Infected pancreatic necrosis can be diagnosed mainly by laboratory methods like culture and radiographic scan. The management of AP has developed considerably during the past decades. The preferred choices are primary conservative and minimally invasive modalities compared to open surgical necrosectomy. Antibiotics are generally not given to patients with severe AP because of limited clinical benefits. Various aspects of infections of AP inclusive of diagnostic and management modalities have been reviewed here.

Keywords: Diagnosis, Infected pancreatic necrosis, Management, Pancreatic infections.

Journal of Gastrointestinal Infections (2019): 10.5005/jp-journals-10068-3030
\end{abstract}

\section{INTRODUCTION}

Acute pancreatitis (AP) is a reversible inflammatory disease of the pancreas with acinar cell destruction ${ }^{1}$ and characterized by pain in abdomen and increased pancreatic enzymes. ${ }^{2}$ Acute pancreatitis is the most unpredictable disease of the digestive system and accounts for more than $50 \%$ of all hospital admissions for pancreatic diseases. ${ }^{3}$ The disease is generally mild and self-limiting. However, about $20 \%$ of the patients develop moderate to severe AP. Severe AP has poor prognosis because of infectious complications and leads to high morbidity, with mortality up to $35 \% .{ }^{4}$ Globally, AP accounts for 1-60 deaths per 100,000 person-years, with an approximate mortality rate of $2-9 \% .^{5,6}$ The reported incidence of AP varies from 12 to 38 per 100,000 population. ${ }^{7,8}$ The indicators of infection include older age group, severity of pancreatitis, and earlier antibiotic use. Significant decrease in mortality can be possible with targeted therapy that can control systemic inflammation.

In recent years, the occurrence of AP has been notably rising, ${ }^{9}$ and because of its impending grave nature, particularly for severe $\mathrm{AP}$, there is an increased claim on healthcare resources, as it requires several weeks to months for treatment. In this review, focus shall be made on various aspects of infections of AP, inclusive of diagnostic methods and commonly used management modalities.

\section{Pathogenesis}

Acute pancreatitis may appear as an interstitial disease or it may be complicated by pancreatic and/or peripancreatic necrosis, which may become infected in up to $40 \%$ of cases. ${ }^{10}$ The pathogenesis of secondary bacterial pancreatic infection is still under controversy. The pathogens can get into the pancreas through several routes such as the hematogenous route, through transmural colonic migration, or through the biliary system. ${ }^{11}$ Acute pancreatitis is the setting for most infections of the pancreas, which can complicate various stages of pancreatitis. About $15-20 \%$ of patients

\footnotetext{
${ }^{1-3}$ Department of Gastroenterology, Postgraduate Institute of Medical Education and Research, Chandigarh, India
}

Corresponding Author: Chetana Vaishnavi, Department of Gastroenterology, Postgraduate Institute of Medical Education and Research, Chandigarh, India, Phone: +91 9417002682, e-mail: cvaishnavi@rediffmail.com

How to cite this article: Vaishnavi C, Bush N, Kochhar R. Infections in Acute Pancreatitis: A Review. J Gastrointest Infect 2019;9(1):28-37.

Source of support: Nil

Conflict of interest: None

develop moderately severe or more severe disease. ${ }^{12}$ In a small group of patients, AP takes a serious course and develops into necrotizing pancreatitis that is characterized by localized or generalized pancreatic parenchymal necrosis and/or necrosis of the retroperitoneal peripancreatic fat. Patients who develop severe AP at an early stage have a high mortality rate. ${ }^{13,14}$

Infected pancreatic necrosis (IPN) is an independent predictor of outcome in AP. ${ }^{15,16}$ The mortality in AP ranges from 4\% to $25 \% .14,15,17$ An important role is played by translocation of microorganisms across the intestinal wall and their virulence, and dysfunction of the lymphocytes. When the pancreatic necrosis becomes infected, mortality rate may go up to $30-32 \% .^{16,18,19}$ There are two incidence peaks of mortality in AP. ${ }^{20}$ The early phase or the first incidence peak occurs within the first week, when the immune system is enormously activated. This phase is associated with up to $50 \%$ of all deaths during the first 2 weeks of illness and mortality occurs due to multiple organ failure. In the late phase or the second incidence peak that can last for weeks to months, the mortality generally occurs due to sepsis. ${ }^{21,22}$ The peak of the infection ranges from the 2 nd to 4 th week after the onset of pancreatitis. ${ }^{23}$ The mean duration of infection is generally after 10 days of illness and peaks during the 2 nd to 4 th week after the onset of pancreatitis. ${ }^{24}$ 
In a meta-analysis of systemic reviews comprising of 6,970 patients, the mortality rate reported was $35.2 \%{ }^{18}$ Jain et al. ${ }^{25}$ in a prospective study in 206 AP patients reported $22 \%$ overall mortality with deaths among $52 \%$ patients with severe AP, particularly in those with multiple organ failure. Higher mortality (42\%) was observed among patients with infected acute necrotic collections compared to infected walled-off necrosis (33\%).

Patients with AP-related IPN frequently develop organ failure and therefore IPN may be associated with increased mortality, ${ }^{16}$ which can be further complicated by multidrug-resistant (MDR) microorganisms. ${ }^{25}$ Thandassery et al. ${ }^{26}$ in their study of 81 AP patients reported hypotension and APACHE II score at 24 hours of hospital admission as independent predictors of occurrence of IPN. The rate of infection correlates with the extent of pancreatic necrosis and its bacterial contamination. The immunologic mechanisms to prevent the proliferation of bacteria and fungi are absent in the necrotic tissue.

\section{Infected Necrosis vs Sterile Necrosis}

Microbial translocation is defined as the passage of both viable and nonviable microbes and microbial products across an anatomically intact intestinal barrier. ${ }^{27}$ Translocation of viable microbes causes sepsis requiring antibiotic prophylaxis whereas nonviable bacterial products like pathogen-associated molecular patterns (PAMPs) cause systematic inflammatory response syndrome (SIRS) where antibiotic use is futile. In about half the number of AP patients, SIRS leads to multiple organ failure and subsequent death.

Clinically, there are no reliable parameters to distinguish infected from sterile necrosis. Microbial PAMPs such as lipopolysaccharides (LPS) activate innate immunocytes through pattern recognition receptors. The immune system also detects "danger," through damage-associated molecular pattern (DAMP) molecules derived from other tissues. Thus, DAMPs stimulate an acute-phase response that is biologically concordant with PAMPs released during infection. This explains why it is difficult to distinguish infectious from noninfectious SIRS or to identify single molecules or molecular patterns of the host response that allow this distinction. Pathogen-associated molecular patterns cause infection when present on viable bacteria. Nonviable bacteria and bacterial products (LPS), which are released spontaneously upon exposure to various triggering factors like bacterial death by antibiotics, can be accompanied by an inflammatory response. ${ }^{28}$ The clinical phenotype of a patient with sepsis may be similar to that of a patient with a SIRS caused by sterile inflammation like pancreatitis. ${ }^{29}$

Bacterial infection of pancreatic necrosis can be seen in 30-50\% of patients of necrotizing pancreatitis. ${ }^{30} \mathrm{Clinical}$ indicators for sepsis like fever and leukocytosis have been found to be higher among patients with IPN. The development of new onset fever, tachycardia, worsening SIRS, or organ failure should prompt evaluation for sepsis and suspicion of infected necrosis. The presence of gas or air bubbles in the collection is highly suggestive of infection but is present only in a minority of cases. ${ }^{31}$ Infection rates progressively increase with duration of the disease with highest incidence in the 3rd week. A direct correlation between extent of necrosis and incidence of infection has been demonstrated in a previous study where $56 \%$ of patients with more than $50 \%$ necrosis had infection as compared to $16.7 \%$ of those with less than $30 \%$ necrosis. ${ }^{32}$

\section{Pancreatic vs Extrapancreatic Infections}

Pancreatic infections may present itself as IPN that occurs earlier in the course of the disease or as infected pseudocysts and pancreatic abscesses that are the sequelae of acute necrotizing pancreatitis occurring more than a month after the acute episode. Infections in severe AP can be pancreatic or systemic and the prevalence usually correlates with the extent of pancreatic necrosis. ${ }^{33,34}$ The probability of super infection of necrosis increases from $24 \%$ in the 1 st week of the illness to $36 \%$ in the 2 nd and $71 \%$ in the 3 rd week. ${ }^{35}$ Patients with extensive necrosis are at greater risk than those with focal necrosis. The main source of infections of the pancreas is direct translocation of bacteria from the large intestine. ${ }^{36-38}$ During such times, the intestinal barrier fails, allowing the organisms to translocate to mesenteric lymph nodes, the systemic circulation, the portal venous circulation, the peritoneal cavity, and abdominal organs, with resulting supervening sepsis and critical complications. This could explain pancreatic infection with Escherichia coli, which is reported to be the most common organism in this setting. ${ }^{39}$ The exact mechanism of bacterial translocation however is controversial and could be via lymphatics and consecutive hematogenous spread, or transmurally to the pancreas. Stress, like acute inflammation, renders the pancreas vulnerable to bacterial infections.

In the majority of patients, AP is generally mild and accompanied by minimal retroperitoneal inflammation and a peripancreatic fluid collection. In fact, AP is the setting for most infections of pancreas, which can complicate various stages of pancreatitis. In about $20 \%$ of the patients, peripancreatic fluid collection may be secondary to disruption of the pancreatic ductal system that later on develops into a pancreatic pseudocyst. ${ }^{35}$ In $20-40 \%$ of patients with severe AP infection, pancreatic and peripancreatic necrosis may occur, leading to deterioration of organ functions. ${ }^{23}$

Infection of the (peri) pancreatic necrosis occurs in about a third of these patients, which leads to $15-39 \%$ mortality rates. ${ }^{5,40-43}$ Half of the mortality occurs later from complications secondary to the infection of (peri) pancreatic necrosis and the ensuing interventions. ${ }^{22,44}$ In a study by Garg et al., ${ }^{39}$ extrapancreatic bacterial infections were found in $31.7 \%$ of 63 patients. In a multicenter audit from Italy, 56 of 161 patients with demonstrated pancreatic necrosis developed infection. ${ }^{45}$ Bourgaux et al. ${ }^{46}$ reported extrapancreatic infections in $25 \%$ of their AP patients. In a group of 713 patients, of whom only 154 had documented pancreatic necrosis, Besselink et al. ${ }^{24}$ reported IPN in 98 patients, yielding an incidence of $63.3 \%$. Petrov et al. ${ }^{16}$ in a meta-analysis of 14 studies comprising of 1,478 AP cases reported IPN in 314 (21\%) patients, with an incidence in the individual reports ranging from $4 \%$ to $63 \%$. Noor et al. ${ }^{32}$ observed pancreatic infections in $37.3 \%$ of patients and extrapancreatic infections in $62.7 \%$ of patients with severe AP with a significant increase in mortality.

Pando et al. ${ }^{47}$ studied the role of extrapancreatic infections in the prediction of severity and local complications in AP and found that extrapancreatic infections played a role in predicting the severity and local complications in AP and when bacteremia and IPN occur, bacteremia preceded infected necrosis in all cases. Grajales-Figueroa et al. ${ }^{48}$ demonstrated increased global mortality rate of 2.5\% (57/2,227 AP patients) from extrapancreatic infections in hospitalized patients with AP over a period of 10 years.

\section{Organisms}

The bacterial spectrum in IPN reflects the commensal flora of the intestine with predominantly gram-negative organisms such as E. coli, Pseudomonas aeruginosa, Proteus spp., and Klebsiella spp. ${ }^{39}$ E. coli is also reported in many other studies to be the most common isolate in pancreatic infections, ${ }^{39,49,50}$ though in one study, Klebsiella was reported to be the most common isolate. ${ }^{51}$ 
However, at times, gram-positive bacteria viz. Staphylococcus aureus, Streptococcus faecalis, Enterococcus, and anaerobes have also been found accountable for pancreatic infections. ${ }^{39,52,53}$ Monomicrobial infections were reported to be more common than polymicrobial. Isenmann et al. ${ }^{13}$ reported a polymicrobial prevalence of $15-40 \%$ among isolates. Furthermore, isolation of polymicrobial spectra should raise suspicion of fistula formation or bowel communication.

A change in the infective spectrum from gram-negative bacteria to that of gram-positive cocci and Candida especially in necrotizing pancreatitis patients on prophylactic antibiotics was reported by Isenmann et al., ${ }^{54}$ which was probably associated with increasing length of hospital stay or related to prophylactic antibiotics administration mainly targeting gram-negative bacteria. ${ }^{24}$ Infections with gram-positive organisms occur later due to nosocomial bloodstream spread. ${ }^{32}$ Noor et al. ${ }^{32}$ studied 51 patients with severe AP and noted pancreatic infection in 19 (37.3\%) patients (14 monomicrobial, 5 polymicrobial) with E. coli as the predominant organism. They also reported 21 positive blood cultures in 16 patients with severe AP and S. aureus was the predominant microorganism followed by E. coli, Enterobacter aerogenes, Alcaligenes xylosoxidans, E. faecalis, P. aeruginosa, Acinetobacter baumannii, and K. pneumonia.

Resistant bacterial isolates were detected in $15.6 \%$ of the necrotic collections in a study by De Waele et al. ${ }^{55}$ However, more recent studies have shown an increasing incidence of MDR organisms in pancreatic infections. Jain et al. ${ }^{25}$ reported culture positivity in $81 \%$ of 108 IPN patients with $86 \%$ growing MDR organisms. They reported E. coli in 48 (32\%) and E. faecium in 10 (7\%) of 149 isolates. Mowbray et al. ${ }^{56}$ investigated the microbiology of 40 IPN patients and reported the most frequently identified microbes as E. faecalis (22.5\%), E. faecium (20.0\%), and E. coli (20.0\%), with 19 cultures being polymicrobial. Sahar et al. ${ }^{53}$ investigated the microbiology of infected walled-off pancreatic necrosis in 182 AP patients of which $41 \%$ were infected. Though MDR organisms was found in only five patients, Candida spp. accounted for $27 \%$. Of those, $55 \%$ were infected, most frequently with Candida spp., Enterococcus spp., and coagulase-negative Staphylococcus. Around $85 \%$ had symptomatic sterile walled-off necrosis treated with minimally invasive therapy, enabling judicious selection of empiric antibiotic use.

Grajales-Figueroa et al. ${ }^{48}$ demonstrated microbiological isolates in IPN in AP patients who died during hospitalization as Staphylococcus spp. (10 no.; 47.6\%), E. faecium (9 no.; $42.8 \%$ ), P. aeruginosa (7 no.; 33.3\%), Candida spp., (6 no.; $28.5 \%)$, and E. coli (5 no.; 23.8\%).

Würstle et al. ${ }^{57}$ analyzed the spectrum of pathogens and their resistance to antibiotics as regards to the time course of the disease and its clinical outcome in 122 patients in Germany. They isolated 309 microorganisms (26 genera, 61 species) viz. Actinomyces spp., Aeromonas spp., Bacteroides spp., Citrobacter spp., Clostridium spp., Corynebacterium spp., Enterobacter spp., Enterococcus spp., Escherichia spp., Fusobacterium spp., Haemophilus spp., Klebsiella spp., Lactobacillus spp., Morganella spp., Neisseria spp., Parvimonas spp., Peptostreptococcus spp., Prevotella spp., Proteus spp., Pseudomonas spp., Rothia spp., Serratia spp., Staphylococcus spp., Stenotrophomonas spp., Streptococcus spp., and Veillonella spp. The authors also observed change at species level of E. faecalis to E. faecium and Candida albicans to non-albicans Candida spp. There was a $59.7 \%$ decrease in antimicrobial susceptibility in the overall number of patients with Enterobacteriaceae and a $46.0 \%$ increase in the incidence of MDR bacteria with increased length of hospital stay. A significantly higher mortality was observed for non-albicans Candida spp., E. faecium, and MDR bacteria suggesting that antimicrobial treatment of IPN becomes more challenging over time, due to an increase of multiresistant difficult-to-treat pathogens leading to worse clinical outcomes.

\section{DiAgnOSIS}

Infected pancreatic necrosis can be diagnosed mainly by laboratory methods as the clinical signs may be sensitive but not specific. ${ }^{58,59}$ Clinically, there are no reliable parameters to distinguish infected from sterile necrosis as the clinical features are indistinguishable from other infectious conditions. Recently Qiu et al. ${ }^{60}$ reported that artificial neural networks can accurately predict intra-abdominal infection in moderately severe and severe AP.

Laboratory methods for diagnosis of pancreatic infections are mentioned below:

\section{Culture}

Identification of microorganisms can be done by culturing the pancreatic and peripancreatic fluid, which reveals the infecting bacteria that originate from the gastrointestinal tract. Isolating organisms from infectious fluid collections gives an opportunity to correlate them with the normal intestinal flora, thus helping confirm their origin. Correlation of pancreatic infection by blood culture will help to determine the hematogenous route of spread of infection. Computed tomography (CT)-guided fine needle aspiration (FNA) of necrosis for Gram staining and culture of the organisms is the diagnostic tool of choice, as it can help the clinicians in selecting the most suitable antibiotics. ${ }^{61}$ A positive culture or Gram's stain of FNA fluid can confirm infected necrosis with a false-negative rate of $20 \%$. In the past, a positive aspirate was thought to mandate surgical intervention. However, subsequent studies have refuted this claim and hence its clinical relevance in today's scenario has diminished. The principle is to avoid early intervention to the maximum and limit tissue or fluid sampling to the time of intervention that is usually late in the course of the disease in order to avoid risk of external contamination and superinfection. Most standard guidelines recommend against this practice and hence should be avoided.

\section{Molecular Methods}

Molecular identification of isolated bacteria and determination of their antibiotic sensitivity require less time than conventional culture and therefore can be useful for diagnosis. It also helps to guide the clinicians about the appropriate antibiotic to be used for a particular patient, and thereby reduce drug resistance and the consequent mortality.

The molecular method for identification of the organisms via polymerase chain reaction (PCR) is better as it is more sensitive and specific, and is therefore the most widely used technique, as the organisms missed during culture can also be picked up by PCR. Polymerase chain reaction may be especially useful when antibiotics are used empirically when routine culture is negative or yield of fluid aspirate is meager. One limitation of this technique lies in its inability to differentiate between viable and nonviable cells and the resulting overestimation of microbial targets. A promising strategy to avoid this issue relies on the use of nucleic acid intercalating dyes, such as propidium monoazide (PMA), as a sample pretreatment prior to $\mathrm{PCR}^{62}$ for live/dead distinction. Propidium monoazide penetrates only cells with compromised 
membranes and once inside the cell, PMA intercalates into the DNA to which it can be covalently cross-linked upon exposure to light. This irreversible DNA modification results in suppression of PCR amplification. ${ }^{62-64}$ Although the dye is membrane-impermeable in viable cells, environmental stresses increase the permeability of PMA into the cell wall in killed bacteria. ${ }^{65}$

\section{CT Scan: Air in Pancreas for Diagnosis}

In some of the patients with severe AP, the presence of gas in the retroperitoneal area signifies infection in the pancreas. ${ }^{58}$ Radiologically contrast-enhanced CT scan is the primary imaging modality for identification of pancreatic necrosis, and the CT severity index can be useful in quantifying the extent of necrosis, which probably correlates with the possibility of developing infection. ${ }^{13,15}$ Air bubbles, if detected within the necrotic pancreas, are indications of infection (Figs 1 and 2). Positive culture from FNA is the gold standard for confirming IPN. However, Gram stain is usually positive in almost all patients with infected necrosis. The CT-guided FNA has a reported sensitivity and specificity exceeding $95 \%$, while ultrasound-guided FNA has sensitivity of $88 \%$ and specificity of $90 \%{ }^{66,67}$

In the majority of patients with mild AP, CT scan is not necessary. However, if there is a deterioration of their symptoms or there is no improvement, CT scan would be required, which can be suspected by clinical and laboratory evaluation. ${ }^{68}$

\section{Procalcitonin as a Marker of Infection}

Investigation of biomarkers like procalcitonin (PCT) will be useful as adjunct diagnosis. Elevated PCT levels are promising markers of infection in necrotizing pancreatitis with reported sensitivity of $75-94 \%$ and specificity of $83-91 \%$ from small studies. ${ }^{69}$ Estimation of PCT may therefore be valuable in predicting the risk of developing IPN. Ji et al. ${ }^{70}$ observed that the post-percutaneous catheter drainage $(P C D)$ serum procalcitonin level might be a respondent factor that is correlated with the necessity of necrosectomy. However, Ivanusa et al. ${ }^{71}$ reported that measurement of procalcitonin for diagnosis of IPN is inadequate and therefore many other new markers of systemic infection introduced into clinical practice such as presepsin, MID-regional pro-adrenomedullinum, CD64 neutrophil index, etc., may be more useful.

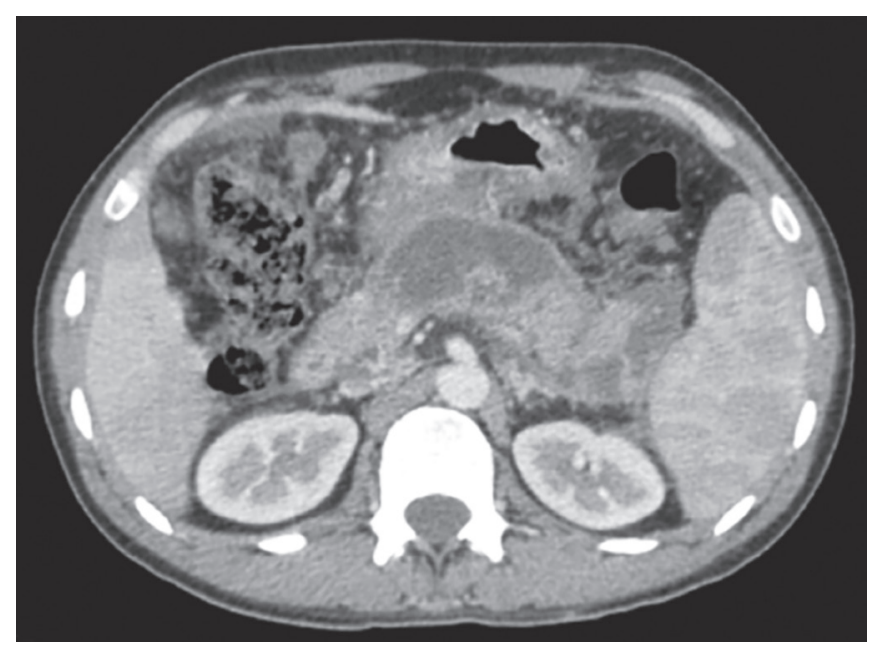

Fig. 1: Contrast-enhanced computed tomography image of acute necrotic collection occupying the peripancreatic area and showing necrosis of $>50 \%$ of the pancreatic parenchyma

\section{Prophylactic Antibiotics}

All standard guidelines do not recommend routine use of prophylactic antibiotics as no significant decrease in mortality and morbidity has been found with them. ${ }^{72-76}$ This is supplemented by multiple meta-analyses that have showed no benefit with antibiotics. ${ }^{76}$ The Japanese guidelines however recommend antibiotics in the severe pancreatitis in the 1st week. Pederzoli et al. ${ }^{77}$ reported that antibiotic administration could prevent infections in patients with sterile necrosis.

Antibiotics are generally not given to patients with severe AP because of limited clinical benefits. However, antibiotics may be useful in some patients with severe AP having infected necrosis but are unfit for surgical debridement and drainage. ${ }^{52}$ Because of the use of antibiotics such as carbapenem and third-generation cephalosporins, MDR microorganisms have increased. ${ }^{78-80}$

The incidence of MDR can be as high as 50\% and microorganisms that are not covered by prophylactic antibiotic regimens become pathogens in subsequent infections. The emergence of MDR may be due to longer duration of antibiotic exposure. Thus, the use of prophylactic antibiotics to prevent pancreatic infection is not recommended. In view of the growing risk of MDR bacteria, antimicrobial prophylaxis has become a real challenge. ${ }^{81,82}$ Lee et al. ${ }^{83}$ reported MDR isolates in 29 of 46 IPN patients with no difference in mortality.

Nakaharai et al. ${ }^{84}$ determined in a population-based study of 3,354 eligible patients in Japan if early prophylactic antibiotics improved the outcomes of severe AP patients and concluded that routine early prophylactic antibiotic use has no significant clinical benefit in severe AP patients but may increase the risk of hospitalacquired infections. Mowbray et al. ${ }^{56}$ reported that antibiotics with the least resistance among the microbiota were teicoplanin (5.0\%), linezolid (5.6\%), ertapenem (6.5\%), and meropenem (7.4\%), and the latter two provide good antimicrobial cover against the common microorganisms causing IPN.

\section{FungaL InFECTIONS}

Recently fungal infections, particularly by Candida, are more and more being identified in severe AP. Risk factors to suspect fungal sepsis in AP include use of broad-spectrum antibiotics, central

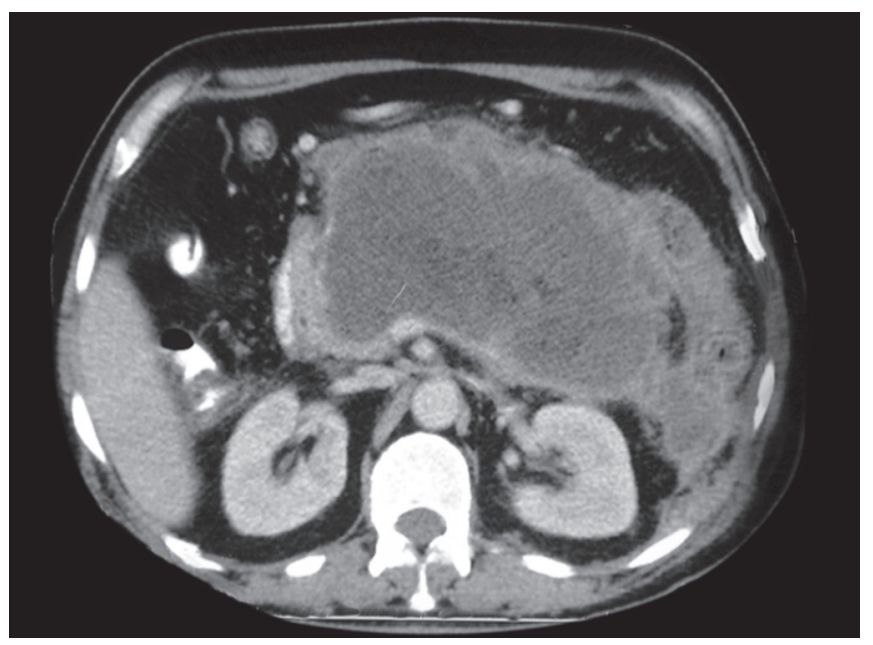

Fig. 2: Contrast-enhanced computed tomography showing walled-off necrosis with scattered air foci suggestive of an infected collection 
venous access, presence of renal failure, high APACHE scores, use of parenteral nutrition, diabetes mellitus, percutaneous drainage, and hospitalization beyond 4 weeks. ${ }^{85}$ This negatively influences the clinical course of the disease with higher incidence of systemic complications leading to morbidity and mortality. ${ }^{54,86-89}$ Demonstration of fungi in biopsy materials is the gold standard of diagnosis of a fungal infection. Grewe et al. ${ }^{90}$ reported an estimation of about $10 \%$ of fungal association in pancreatic necrosis. Even though no significant mortality difference was reported by Gloor et al., ${ }^{91}$ the authors suggest that the incidence could be increased multifold in recent years in hospitals where prophylactic antibiotics are routinely being used. ${ }^{91}$ De Waele et al. ${ }^{92}$ analyzed the association of fungal infections in an 8-year period data for 46 patients with severe AP and IPN and found intra-abdominal fungal infection in 17 (37\%) of them with C. albicans in 15 patients and C. tropicalis and C. krusei in 1 patient each.

The pathogenesis of fungal infection in AP patients is multifactorial. Several reasons could attribute for increased incidence of fungal infection in AP, particularly increased use of broad-spectrum antibiotics ${ }^{54}$ and the severity of the disease. ${ }^{92}$ Candida infection of $37 \%$ was reported by De Waele et al..$^{92}$ and if patients who received early treatment were excluded, the incidence of Candida infection was as high as 50\%.

Among fungal infections, Candida spp. is responsible for about $15-30 \%$ of pancreatic infections. The most common fungi are C. albicans, followed by C. tropicalis and C. krusei. ${ }^{89}$ Jain et al. ${ }^{25}$ observed fungal infection in $13 \%$ (27/209) of patients. Horibe et al., ${ }^{93}$ in a large multicenter cohort comprising of 44 institutions, investigated the impact of antibiotic prophylaxis for severe AP on the development of invasive pancreatic candidiasis. They observed that $1.9 \%$ of the 1,097 patients with severe AP had invasive pancreatic candidiasis and antimicrobial prophylaxis was significantly associated with the development of invasive pancreatic candidiasis. Würstle et al. ${ }^{57}$ reported presence of yeasts in $41.0 \%$ of 122 acute necrotizing pancreatitis patients with C. albicans in $27.9 \%$, whereas the incidence of non-albicans Candida spp. was only $13.9 \%$.

\section{Role of Antifungal Treatment}

There is a lot of controversy regarding the role of antifungal treatment in AP. The risk factors for fungal infection in AP patients comprise of prolonged hospitalization, invasive methods, the use of broad-spectrum antibiotics, total parenteral nutrition, and mechanical ventilation. The risk associated with antifungal treatment, apart from the cost factor, is the selection of non-albicans species of Candida $a^{92}$ with decreased susceptibility or resistance to fluconazole and the induction of resistance in previously susceptible strains of C. albicans. ${ }^{94}$ De Waele et al. ${ }^{92}$ reported development of C. krusei infection after prolonged fluconazole treatment in one patient. Thus, the use of antifungal treatment should be carefully considered for each patient, bearing in mind the potential drawbacks of apparently harmless prophylaxis.

However, antifungal treatment has been recommended for high-risk surgical patients due to the apparent increased mortality and morbidity after surgery. Antifungal prophylaxis with fluconazole was associated with a lower incidence of intraabdominal fungal infection, ${ }^{86,95}$ though it is not clear whether this data can be extrapolated to patients with severe AP. Pelz et al. ${ }^{96}$ studied the efficacy of enteral fluconazole $(400 \mathrm{mg})$ prophylaxis in 260 high-risk patients and reported a 55\% reduction in the risk of acquiring fungal infection in the treatment group, though parenteral administration is advisable to ensure systemic efficacy.

De Waele et al. ${ }^{92}$ reported that though the characteristics of patients with and without fungal infections as well as the mortality rate were insignificant, early treatment with fluconazole could prevent fungal infection in their patients.

\section{Antibiotic Treatment}

The spectrum of pathogens isolated from the IPN helps to decide the choice of antibiotics. Most of the bacteria are sensitive to beta lactam antibiotics, aminoglycosides, and imipenem whereas application of broad-spectrum antibiotics bears the risk for a patient of AP to develop deep fungal infections. ${ }^{97}$ Antimicrobial susceptibility test of selected microorganism helps determine the possible drug resistance in common pathogens and helps to assure susceptibility to drugs of choice for infections. Principles of antimicrobial stewardship include noticing changed pharmacokinetics in the critically ill, decreasing broad-spectrum therapy upon receiving culture reports, and early removal of antibiotics after establishing the source control. This will help to avoid the development of antimicrobial resistance, particularly in patients who may need frequent courses of antibiotics during the course of their disease.

Antibiotic therapy is indicated if pancreatic necrosis is suspected or confirmed on imaging. These patients have a $20-40 \%$ risk of superinfection in the retroperitoneum. The spectrum of pathogens isolated from the IPN helps to decide the choice of antibiotics. Another important deciding factor is the penetration of the antibiotic in the pancreas as selective uptake of various different classes of antibiotics occurs in the pancreas. Adequate tissue concentration in the pancreas occurs with fluoroquinolones, carbapenems, and metronidazole whereas ampicillin and gentamicin do not attain effective therapeutic levels in the pancreatic tissue. Metronidazole is suitable only as an adjunct agent for additional anaerobic coverage. The recommended antibiotics are either a combination of a quinolone (ciprofloxacin or ofloxacin) with metronidazole or, alternatively, monotherapy with imipenem-cilastatin.

In cases of infected AP, antibiotics are always recommended to treat the patients. Acylureidopenicillins and third-generation cephalosporins are effective against gram-negative microorganisms found in pancreatic infections. ${ }^{98}$ However, for gram-positive organisms and anaerobes, only piperacillin/tazobactam is effective. Quinolones (ciprofloxacin and moxifloxacin) and carbapenems have the extra advantage of excellent anaerobic coverage apart from good tissue diffusion into the pancreas. ${ }^{99}$ In terms of tissue penetration, carbapenems have the highest efficacy followed by fluoroquinolones and aminoglycosides. Hence, either of them is preferred for initial management. Appropriate application of antimicrobial treatment is required for treatment of severe AP, which is marked by organ failure and (peri) pancreatic necrosis with local complications such as infected necrosis. Leppäniemi et al. ${ }^{23}$ in their guidelines for the management of severe AP suggest that quinolones should be used only in patients with allergy to betalactams because of their high rate of resistance and carbapenams should be used only in very critically ill patients after optimization due to dissemination of carbapenem-resistant K. pneumonia. The bactericidal antibiotic metronidazole also is capable of penetration into the pancreatic tissue. However, standard intravenous dosages of aminoglycoside antibiotics like gentamicin 
and tobramycin are unable to penetrate into the pancreas in required concentrations. ${ }^{100}$

Longer duration of antimicrobial treatment in patients with severe AP is done in order to delay surgery. However, when the antimicrobial treatment is prolonged, there is a risk for consequent fungal infection or antibiotic resistance. Jain et al. ${ }^{25}$ observed that among those who received prophylactic antibiotics, the rate of isolation of MDR organisms was not significantly different from those who did not receive prophylactic antibiotics.

\section{Other Modes of Management}

The management of AP has developed considerably during the past decades. The preferred choices are primary conservative and minimally invasive modalities compared to open surgical necrosectomy ${ }^{101-103}$ with decreased mortality. ${ }^{104}$ In patients who are asymptomatic, strong suspicion of IPN would require interventions, even though some of them may recover with antibiotics only. ${ }^{105}$ Wolbrink et al. ${ }^{106}$ advocated invasive diagnostics (e.g., through FNA) for management of IPN in the intensive care unit, prior to the start of broad-spectrum antimicrobial therapy. Surgical necrosectomy with antifungal prophylaxis is the most widely used treatment modality.

\section{Endoscopic or Surgical Treatment}

Patients with intra-abdominal-infected necrosis generally receive endoscopic or surgical treatment, which results in an extended hospital stay. ${ }^{107}$ Walled-off necrosis or pseudocysts, when they do not resolve, need an intervention. In a report of 178 cases with walled-off necrosis, $96 \%$ of the patients were subjected to surgical transgastric necrosectomy, with only $2 \%$ postoperative mortality and $38 \%$ morbidity. ${ }^{108}$ It is also a good option in patients with a disconnected duct syndrome.

\section{Percutaneous Catheter Drainage}

Open surgery is regarded to cause a more severe inflammatory response. Percutaneous catheter drainage of IPN allows delaying the later possible surgical intervention to a more favorable time. ${ }^{23}$ In a systematic review consisting of 11 studies and 384 patients, Diaz et al. ${ }^{109}$ reported PCD as primary treatment for necrotizing pancreatitis. Of these, $71 \%$ had IPN and $56 \%$ did not require surgery after PCD. Percutaneous drainage is required when a patient deteriorates. ${ }^{110,111}$ Large-bore catheters appear to be less often obstructed by the necrotic debris and therefore are more beneficial. $^{112}$

If PCD does not result in resolution of the infection, then other options such as open surgery, mini-invasive surgery, endoscopic surgery, or a combination of these can be tried. Shenvi et al. ${ }^{113}$ showed that PCD along with antibiotics was a successful line of treatment in $50 \%$ of IPN patients. Moreover, a study by Jain et al. ${ }^{25}$ in 10 patients showed that PCD prior to referral did not influence the development of MDR infection.

\section{Necrosectomy}

Necrosectomy can be done when the patient does not recover with drainage alone. Open necrosectomy has been largely replaced by minimally invasive techniques such as sinus tract endoscopy and video-assisted retroperitoneal debridement, ${ }^{114}$ which show decreased morbidity and mortality, especially in patients with high risk. ${ }^{107}$ An endoscopic approach to necrosectomy drastically reduces the proinflammatory response with a success rate of about $81 \%{ }^{115}$ but requires repeated sessions for definitive treatment. Ji et al. ${ }^{70}$ assessed the need of surgical necrosectomy after PCD for IPN in 329 patients enrolled and concluded that both mean CT density of necrotic fluid collection and multiple organ failure are independent pre- and post-PCD risk factors for the need of necrosectomy after PCD.

\section{Nutritional Therapies}

Feeding by the enteric route sustains the intestinal mucosal barrier and stops the translocation of bacteria that causes pancreatic necrosis. ${ }^{23}$ It also decreases infectious complications, organ failure, and mortality as compared to total parental nutrition. ${ }^{116}$ Most institutions prefer continuous infusion over cyclic or bolus administration. ${ }^{23}$ Hsieh et al. ${ }^{117}$ reported that nutritional therapy via the nasogastric route was the most preferred therapy for AP whereas total parental nutrition was the least preferred route.

\section{Role of Probiotics}

Initially in several experimental studies, the prophylactic use of probiotics in AP was investigated. van Minnen et al. ${ }^{118}$ reported reduced overgrowth of potential pathogens, decrease in bacterial translocation, and reduced mortality with probiotics use in rats with AP. There were two randomized controlled trials (RCTs) from Hungary regarding use of probiotics in AP. The first trial in 45 AP patients showed that probiotics reduced pancreatic sepsis and the need for surgical intervention. ${ }^{119}$ In the second trial with 62 severe AP patients, Olah et al. ${ }^{120}$ concluded that nasojejunal feeding with synbiotics (inulin, beta-glucan, resistant starch, and pectin) may prevent organ dysfunction in severe AP.

Karakan et al. ${ }^{121}$ reported that supplementation of prebiotic fiber alone with enteral nutrition assessed in a randomized, doubleblind study with 30 severe AP patients showed reduction of hospital stay, period of nutrition therapy, as well as overall complications compared to the usual enteral nutrition therapy. Besselink et al. ${ }^{122}$ reported a multicenter RCT where probiotics were compared with placebo in 298 patients with predicted severe AP. Probiotics were not found to reduce infectious complications, but rather increased the rate of bowel ischemia ( $9 \%$ vs $0 \%$ ) and mortality (16\% vs $6 \%$ ) in the probiotics group. However, Qin et al. ${ }^{123}$ reported that the patients with probiotic therapy showed less disease severity, improved gut permeability, and better clinical results.

Cui et al. ${ }^{124}$ compared parenteral nutrition and enteral nutrition and the latter with the addition of the probiotic Bifidobacterium in 70 severe AP patients and reported that early enteral nutrition with Bifidobacterium resulted in reduced pro-inflammatory cytokines, improved gastrointestinal function, reduced complications, and shorter hospital stay. Gou et al. ${ }^{125}$ studied the use of probiotics in severe AP by including six relevant human RCTs in which a total of 14 strains of probiotic bacteria were used. In total, Lactobacillus plantarum was used in three trials: B. longum, L. bulgaricus, $L$. paracasei, L. mesenteroides, and $P$. pentosaceus were used in different combinations in two trials, and B. bifidum, B. lactis, $E$. faecalis, L. acidophilus, L. casei, L. lactis, L. salivarius, and Streptococcus thermophilus used in just one trial. The authors concluded that probiotics showed neither beneficial nor adverse effects on the clinical outcomes of patients with predicted severe AP. But Wu et al. ${ }^{126}$ investigated 120 patients with severe AP and found that probiotics could play a useful role in the treatment of severe AP. Bouwense et al. ${ }^{127}$ observed that currently there was no place for probiotics 
as a treatment for AP. As there was a heterogeneity in the types of probiotics and treatment strategies used, even though previous data imply the potential of single specific probiotic strains supplemented, further appraisal about the role of probiotics is required. ${ }^{128}$

\section{Conclusion}

A high index of suspicion is required for prompt diagnosis of pancreatic infections as early diagnosis and treatment can result in improved outcomes. Strict adherence to a rational antimicrobial treatment for patients with necrotizing AP is quite important. The antimicrobial therapy of IPN is more challenging with the length of the disease. Therefore, these patients can be managed well with appropriate diagnosis and identification of infection in order to improve the clinical outcome so that a change to narrow-spectrum antimicrobials can be done. Unnecessary antibiotic therapy without apparent proof of persisting infection should be avoided, as every antimicrobial treatment can lead to resistance, thereby complicating the treatment further. Other important preventive strategies include introduction of enteral nutrition and periodic change of vascular catheters. Furthermore, indications for surgical intervention should be defined and clinical trials on antifungal treatment are required.

\section{References}

1. Garber A, Frakes C, Arora Z, et al. Mechanisms and management of acute pancreatitis. Gastroenterol Res Pract 2018;2018:6218798. DOI: 10.1155/2018/6218798.

2. Banks PA, Bollen TL, Dervenis C, et al. Classification of acute pancreatitis--2012: revision of the Atlanta classification and definitions by international consensus. Gut 2013;62(1):102-111. DOI: 10.1136/gutjnl-2012-302779.

3. NICE guideline. Pancreatitis diagnosis and management. March 2018. Available from: www.nice.org.uk.

4. van Dijk SM, Hallensleben NDL, van Santvoort HC, et al. Acute pancreatitis: recent advances through randomised trials. Gut 2017;66(11):2024-2032. DOI: 10.1136/gutjnl-2016-313595.

5. Banks PA, Freeman ML, Practice Parameters Committee of the American College of Gastroenterology. Practice guidelines in acute pancreatitis. Am J Gastroenterol 2006;101(10):2379-2400. DOI: 10.1111/j.1572-0241.2006.00856.x.

6. Xiao AY, Tan MLY, Wu LM, et al. Global incidence and mortality of pancreatic diseases: a systematic review, meta-analysis, and metaregression of population-based cohort studies. Lancet Gastroenterol Hepatol 2016;1(1):45-55. DOI: 10.1016/S2468-1253(16)30004-8.

7. Eland IA, Sturkenboom MC, van der Lei J, et al. Incidence and mortality of acute pancreatitis between 1985 and 1995. Scand J Gastroenterol 2000;35(10):1110-1116. DOI: 10.1080/003655200451261.

8. Frey CF, Zhou H, Harvey DJ, et al. The incidence and case-fatality rates of acute biliary, alcoholic, and idiopathic pancreatitis in California, 1994-2001. Pancreas 2006;33(4):336-344. DOI: 10.1097/01. mpa.0000236727.16370.99.

9. Yadav D, Lowenfels AB. The epidemiology of pancreatitis and pancreatic cancer. Gastroenterology 2013;144(6):1252-1261. DOI: 10.1053/j.gastro.2013.01.068.

10. Uhl W, Warshaw A, Imriec C, et al. IAP Guidelines for the surgical management of acute pancreatitis. Pancreatology 2002;2(6):565-573. DOI: 10.1159/000067684.

11. Mourad MM, Evans R, Kalidindi V, et al. Prophylactic antibiotics in acute pancreatitis: endless debate. Ann R Coll Surg Engl 2017;99(2):107-112. DOI: 10.1308/rcsann.2016.0355.

12. Sarri G, Guo Y, Iheanacho I, et al. Moderately severe and severe acute pancreatitis: a systematic review of the outcomes in the USA and European Union-5. BMJ Open Gastroenterol 2019;6(1):e000248. DOI: 10.1136/bmjgast-2018-000248.
13. Isenmann R, Rau B, Beger HG. Bacterial infection and extent of necrosis are determinants of organ failure in patients with acute necrotizing pancreatitis. Br J Surg 1999;86(8):1020-1024. DOI: 10.1046/j.1365-2168.1999.01176.x.

14. Sharma M, Banerjee D, Garg PK. Characterization of newer subgroups of fulminant and subfulminant pancreatitis associated with a high early mortality. Am J Gastroenterol 2007;102(12):2688-2695. DOI: 10.1111/j.1572-0241.2007.01446.x.

15. Garg PK, Madan K, Pande GK, et al. Association of extent and infection of pancreatic necrosis with organ failure and death in acute necrotizing pancreatitis. Clin Gastroenterol Hepatol 2005;3(2): 159-166. DOI: 10.1016/S1542-3565(04)00665-2.

16. Petrov MS, Shanbhag $S$, Chakraborty $M$, et al. Organ failure and infection of pancreatic necrosis as determinants of mortality in patients with acute pancreatitis. Gastroenterology 2010;139(3): 813-820. DOI: 10.1053/j.gastro.2010.06.010.

17. Bota S, Sporea I, Sirli R, et al. Predictive factors for severe evolution in acute pancreatitis and a new score for predicting a severe outcome. Ann Gastroenterol 2013;26(2):156-162.

18. Werge M, Novovic S, Schmidt PN, et al. Infection increases mortality in necrotizing pancreatitis: a systematic review and meta-analysis. Pancreatology 2016;16(5):698-707. DOI: 10.1016/j.pan.2016.07.004.

19. Boumitri C, Brown E, Kahaleh M. Necrotizing pancreatitis: current management and therapies. Clin Endosc 2017;50(4):357-365. DOI: 10.5946/ce.2016.152.

20. Algül HAW, Huber W, Schmid RM. Acute pancreatitis. In: Podolsky DK, Fitz JG, Kalloo AN, et al. ed. Yamada's Textbook of Gastroenterology. Publishers John Wiley \& Sons; 2015. p. 82.

21. Blum T, Maisonneuve $P$, Lowenfels $A B$, et al. Fatal outcome in acute pancreatitis: its occurrence and early prediction. Pancreatology 2001;1(3):237-241. DOI: 10.1159/000055817.

22. Carnovale A, Rabitti PG, Manes G, et al. Mortality in acute pancreatitis: is it an early or a late event? JOP 2005;6(5):438-444.

23. Leppäniemi A, Tolonen M, Tarasconi A, et al. 2019 WSES guidelines for the management of severe acute pancreatitis. World J Emerg Surg 2019;14:27. DOI: 10.1186/s13017-019-0247-0.

24. Besselink MG, van Santvoort HC, Boermeester MA, et al. Timing and impact of infections in acute pancreatitis. Br J Surg 2009;96(3): 267-273. DOI: 10.1002/bjs.6447.

25. Jain S, Mahapatra SJ, Gupta S, et al. Infected pancreatic necrosis due to multidrug-resistant organisms and persistent organ failure predict mortality in acute pancreatitis. Clin Transl Gastroenterol 2018;9(10):190. DOI: 10.1038/s41424-018-0056-x.

26. Thandassery RB, Yadav TD, Dutta U, et al. Hypotension in the first week of acute pancreatitis and APACHE II score predict development of infected pancreatic necrosis. Dig Dis Sci 2015;60(2):537-542. DOI: 10.1007/s10620-014-3081-y.

27. Novati S, Sacchi P, Cima S, et al. General issues on microbial translocation in HIV-infected patients. Eur Rev Med Pharmacol Sci 2015;19(5):866-878.

28. Evans ME, Pollack M. Effect of antibiotic class and concentration on the release of lipopolysaccharide from Escherichia coli. J Infect Dis 1993;167(6):1336-1343. DOI: 10.1093/infdis/167.6.1336.

29. Matzinger $P$. The danger model: a renewed sense of self. Science 2002;296(5566):301-305. DOI: 10.1126/science.1071059.

30. Bassi C, Falconi M, Girelli R, et al. Microbiological findings in severe acute pancreatitis. Surg Res Commun 1989;5(1):1-4.

31. Shyu JY, Sainani NI, Sahni VA, et al. Necrotizing pancreatitis: diagnosis, imaging, and intervention. Radiographics 2014;34(5):1218-1239. DOI: 10.1148/rg.345130012.

32. Noor MT, Radhakrishna Y, Kochhar R, et al. Bacteriology of infection in severe acute pancreatitis. JOP 2011;12(1):19-25.

33. Khan A, Khan S. Antibiotics in acute necrotizing pancreatitisperspective of a developing country. J Pak Med Assoc 2010;60(2): 121-126.

34. Babu RY, Gupta R, Kang M, et al. Predictors of surgery in patients with severe acute pancreatitis managed by the step up approach. Ann Surg 2013;257(4):737-750. DOI: 10.1097/SLA.0b013e318269d25d. 
35. Buchler MW, UhI W, Malfertheiner P, et al. Acute pancreatitis, chronic pancreatitis, neoplasms of the pancreas. Diseases of the Pancreas. Basel: Karger Publishers; 2004.

36. Schmid SW, Uhl W, Friess $\mathrm{H}$, et al. The role of infection in acute pancreatitis. Gut 1999;45(2):311-316. DOI: 10.1136/gut.45.2.311.

37. Liu H, Li W, Wang X, et al. Early gut mucosal dysfunction in patients with acute pancreatitis. Pancreas 2008;36(2):192-196. DOI: 10.1097/ MPA.0b013e31815a399f.

38. Vaishnavi C. Translocation of gut flora and its role in sepsis. Indian J Med Microbiol 2013;31(4):334-342. DOI: 10.4103/0255-0857.118870.

39. Garg PK, Khanna S, Bohidar NP, et al. Incidence, spectrum and antibiotic sensitivity pattern of bacterial infections among patients with acute pancreatitis. J Gastroenterol Hepatol 2001;16(9):1055-1059. DOI: 10.1046/j.1440-1746.2001.02589.x.

40. Traverso LW, Kozarek RA. Pancreatic necrosectomy: definitions and technique. J Gastrointest Surg 2005;9(3):436-439. DOI: 10.1016/ j.gassur.2004.05.013.

41. Raraty MG, Halloran CM, Dodd S, et al. Minimal access retroperitoneal pancreatic necrosectomy: improvement in morbidity and mortality with a less invasive approach. Ann Surg 2010;251(5):787-793. DOI: 10.1097/SLA.0b013e3181d96c53.

42. Freeman ML, Werner J, van Santvoort HC, et al. Interventions for necrotizing pancreatitis: summary of a multidisciplinary consensus conference. Pancreas 2012;41(8):1176-1194. DOI: 10.1097/ MPA.0b013e318269c660.

43. Tan V, Charachon A, Lescot T, et al. Endoscopic transgastric versus surgical necrosectomy in infected pancreatic necrosis. Clin Res Hepatol Gastroenterol 2014;38(6):770-776. DOI: 10.1016/ j.clinre.2014.06.016.

44. Fu CY, Yeh CN, Hsu JT, et al. Timing of mortality in severe acute pancreatitis: experience from 643 patients. World J Gastroenterol 2007;13(13):1966-1969. DOI: 10.3748/wjg.v13.i13.1966.

45. Pezzilli R, Uomo G, Gabbrielli A, et al. A prospective multicentre survey on the treatment of acute pancreatitis in Italy. Dig Liver Dis 2007;39(9):838-846. DOI: 10.1016/j.dld.2007.05.014.

46. Bourgaux JF, Defez C, Muller L, et al. Infectious complications, prognostic factors and assessment of anti infectious management of 212 consecutive patients with acute pancreatitis. Gastroenterol Clin Biol 2007;31(4):431-435. DOI: 10.1016/S0399-8320(07)89406-1.

47. Pando $E$, Alberti $P$, Hidalgo J, et al. The role of extra-pancreatic infections in the prediction of severity and local complications in acute pancreatitis. Pancreatology 2018; S1424-3903(18)30587-8. DOI: 10.1016/j.pan.2018.05.481.

48. Grajales-Figueroa G, Hernández HAD, Portillo MAC, et al. Increased mortality from extrapancreatic infections in hospitalized patients with acute pancreatitis. Gastroenterol Res Pract 2019;2019:2789764. DOI: $10.1155 / 2019 / 2789764$.

49. Beger HG, Bittner R, Block $S$, et al. Bacterial contamination of pancreatic necrosis: a prospective clinical study. Gastroenterology 1986;91(2):433-438. DOI: 10.1016/0016-5085(86)90579-2.

50. Bradley 3rd EL. A fifteen year experience with open drainage for infected pancreatic necrosis. Surg Gynecol Obstet 1993;177(3): 215-222.

51. Gerzof SG, Banks PA, Robbins AH, et al. Early diagnosis of pancreatic infection by computed tomography-guided aspiration. Gastroenterology 1987;93(6):1315-1320. DOI: 10.1016/00165085(87)90261-7.

52. Rau B, Bothe A, Beger HG. Surgical treatment of necrotizing pancreatitis by necrosectomy and closed lavage: changing patient characteristics and outcome in a 19-year, single-center series. Surgery 2005;138(1):28-39. DOI: 10.1016/j.surg.2005.03.010.

53. Sahar N, Kozarek RA, Kanji ZS, et al. The microbiology of infected pancreatic necrosis in the era of minimally invasive therapy. Eur J Clin Microbiol Infect Dis 2018;37(7):1353-1359. DOI: 10.1007/s10096018-3259-x.

54. Isenmann R, Schwarz M, Rau B, et al. Characteristics of infection with candida species in patients with necrotizing pancreatitis. World $J$ Surg 2002;26(3):372-376. DOI: 10.1007/s00268-001-0146-9.
55. De Waele JJ, Vogelaers D, Hoste E, et al. Emergence of antibiotic resistance in infected pancreatic necrosis. Arch Surg 2004;139(12): 1371-1375. DOI: 10.1001/archsurg.139.12.1371.

56. Mowbray NG, Ben-Ismaeil B, Hammoda M, et al. The microbiology of infected pancreatic necrosis. Hepatobiliary Pancreat Dis Int 2018;17(5):456-460. DOI: 10.1016/j.hbpd.2018.08.007.

57. Würstle $S$, Hapfelmeier A, Wöhrle $C$, et al. Changes in pathogen spectrum and antimicrobial resistance development in the timecourse of acute necrotizing pancreatitis. J Gastroenterol Hepatol 2019;34(12):2096-2103. DOI: 10.1111/jgh.14748.

58. De Waele JJ. Rational use of antimicrobials in patients with severe acute pancreatitis. Semin Respir Crit Care Med 2011;32(2):174-180. DOI: $10.1055 / \mathrm{s}-0031-1275529$.

59. Stigliano S, Sternby H, de Madaria E, et al. Early management of acute pancreatitis: a review of the best evidence. Dig Liver Dis 2017;49(6):585-594. DOI: 10.1016/j.dld.2017.01.168.

60. Qiu Q, Nian YJ, Tang L, et al. Artificial neural networks accurately predict intra-abdominal infection in moderately severe and severe acute pancreatitis. J Dig Dis 2019;20(9):486-494. DOI: 10.1111/17512980.12796.

61. Guarner-Argente C, Shah P, Buchner A, et al. Use of antimicrobials for EUS-guided FNA of pancreatic cysts: a retrospective, comparative analysis. Gastrointest Endosc 2011;74(1):81-86. DOI: 10.1016/ j.gie.2011.03.1244.

62. Nocker A, Cheung CY, Camper AK. Comparison of propidium monoazide with ethidium monoazide for differentiation of live vs dead bacteria by selective removal of DNA from dead cells. J Microbiol Methods 2006;67(2):310-320. DOI: 10.1016/j.mimet.2006. 04.015 .

63. Nogva HK, Drømtorp SM, Nissen H, et al. Ethidium monoazide for DNA-based differentiation of viable and dead bacteria by 5 '-nuclease PCR. Biotechniques 2003;34(4):804-813. DOI: 10.2144/03344rr02.

64. Nocker A, Sossa KE, Camper AK. Molecular monitoring of disinfection efficacy using propidiummonoazide in combination with quantitative PCR. J Microbiol Methods 2007;70(2):252-260. DOI: 10.1016/j.mimet.2007.04.014.

65. Pribylova R, Kubickova L, Babak V, et al. Effect of short- and long-term antibiotic exposure on the viability of Mycobacterium avium subsp. paratuberculosis as measured by propidium monoazide F57 real time quantitative PCR and culture. Vet J 2012;194(3):354-360. DOI: 10.1016/j.tvjl.2012.05.002.

66. Banks PA, Gerzof SG, Langevin RE, et al. CT-guided aspiration of suspected pancreatic infection: bacteriology and clinical outcome. Int J Pancreatol 1995;18(3):265-270. DOI: 10.1007/BF02784951.

67. Rau B, Pralle U, Mayer JM, et al. Role of ultrasonographically guided fine-needle aspiration cytology in the diagnosis of infected pancreatic necrosis. Br J Surg 1998;85(2):179-184. DOI: 10.1046/j.13652168.1998.00707.x.

68. Working Group IAP/APA Acute Pancreatitis Guidelines. IAP/ APA evidence based guidelines for the management of acute pancreatitis. Pancreatology 2013;13(4 Suppl 2):e1-e15. DOI: 10.1016/ j.pan.2013.07.063.

69. Mofidi R, Suttie SA, Patil PV, et al. The value of procalcitonin at predicting the severity of acute pancreatitis and development of infected pancreatic necrosis: systematic review. Surgery 2009;146(1):72-81. DOI: 10.1016/j.surg.2009.02.013.

70. JiL, Wang G, LiL, etal. Riskfactors for the need of surgical necrosectomy after percutaneous catheter drainage in the management of infection secondary to necrotizing pancreatitis pancreas. Pancreas 2018;47(4):436-443. DOI: 10.1097/MPA.0000000000001031.

71. Ivanusa SY, Ivanov AM, Lazutkin MV, et al. Opportunities of modern laboratory diagnostics of infectious complications of acute pancreatitis (review). Klin Lab Diagn 2019;64(3):145-152. DOI: 10.18821/0869-2084-2019-64-3-145-152.

72. Isenmann R, Runzi M, Kron M, et al. Prophylactic antibiotic treatment in patients with predicted severe acute pancreatitis: a placebocontrolled, double blind trial. Gastroenterology 2004;126(4): 997-1004. DOI: 10.1053/j.gastro.2003.12.050. 
73. Dellinger EP, Tellado JM, Soto NE, et al. Early antibiotic treatment for severe acute necrotizing pancreatitis: randomized, double-blind, placebo-controlled study. Ann Surg 2007;245(5):674-683. DOI: 10.1097/01.sla.0000250414.09255.84.

74. Sakorafas GH, Lappas C, Mastoraki A, et al. Current trends in the management of infected necrotizing pancreatitis. Infect Disord Drug Targets 2010;10(1):9-14. DOI: 10.2174/187152610790410936.

75. Villatoro E, Mulla M, Larvin M. Antibiotic therapy for prophylaxis against infection of pancreatic necrosis in acute pancreatitis. Cochrane Database Syst Rev 2010;5:CD002941.DOI: 10.1002/14651858. CD002941.pub3.

76. Jiang K, Huang W, Yang XN, et al. Present and future of prophylactic antibiotics for severe acute pancreatitis. World J Gastroenterol 2012;18(3):279-284. DOI: 10.3748/wjg.v18.i3.279.

77. Pederzoli P, Bassi C, Vesentini S, et al. A randomized multicenter clinical trial of antibiotic prophylaxis of septic complications in acute necrotizing pancreatitis with imipenem. Surg Gynecol Obstet 1993;176(5):480-483.

78. Sabharwal ER. Antibiotic susceptibility patterns of uropathogens in obstetric patients. N Am J Med Sci 2012;4(7):316-319. DOI: 10.4103/1947-2714.98591.

79. Joshi S, Ray P, Manchanda V, et al. Methicillin resistant Staphylococcus aureus (MRSA) in India: prevalence \& susceptibility pattern. Indian J Med Res 2013;137(2):363-369.

80. Lim C, Takahashi E, Hongsuwan M, et al. Epidemiology and burden of multidrug-resistant bacterial infection in a developing country. eLife 2016;5:e18082. DOI: 10.7554/eLife.18082.

81. Peirano G, van der Bij AK, Gregson DB, et al. Molecular epidemiology over an 11-year period (2000 to 2010) of extended-spectrum betalactamase-producing Escherichia coli causing bacteremia in a centralized Canadian region. J Clin Microbiol 2012;50(2):294-299. DOI: 10.1128/JCM.06025-11.

82. Pitout JD. Enterobacteriaceae that produce extended-spectrum beta-lactamases and AmpC beta-lactamases in the community: the tip of the iceberg? Curr Pharm Des 2013;19(2):257-263. DOI: $10.2174 / 138161213804070348$.

83. Lee HS, Lee SK, Park DH, et al. Emergence of multidrug resistant infection in patients with severe acute pancreatitis. Pancreatology 2014;14(6):450-453. DOI: 10.1016/j.pan.2014.10.003.

84. Nakaharai K, Morita K, Jo T, et al. Early prophylactic antibiotics for severe acute pancreatitis: a population-based cohort study using a nationwide database in Japan. J Infect Chemother 2018;24(9): 753-758. DOI: 10.1016/j.jiac.2018.05.009.

85. Kochhar R, Noor MT, Wig J. Fungal infections in severe acute pancreatitis. J Gastroenterol Hepatol 2011;26(6):952-959. DOI: 10.1111/j.1440-1746.2011.06685.x.

86. Hoerauf A, Hammer S, Muller-Myhsok B, et al. Intra-abdominal candida infection during acute necrotizing pancreatitis has a high prevalence and is associated with increased mortality. Crit Care Med 1998;26(12):2010-2015. DOI: 10.1097/00003246-199812000-00031.

87. Kujath $P$, Rosenfeldt $M$, Esnaashari $H$, et al. Fungal infections in patients with necrotizing pancreatitis: risk-factors, incidence, therapy. Mycoses 2005;48(Suppl 1):36-40. DOI: 10.1111/j.14390507.2005.01108.x.

88. Schwender BJ, Gordon SR, GardnerTB. Riskfactors for the development of intra-abdominal fungal infections in acute pancreatitis. Pancreas 2015;44(5):805-807. DOI: 10.1097/MPA.0000000000000334.

89. Reuken PA, Albig $\mathrm{H}$, Rödel J, et al. Fungal infections in patients with infected pancreatic necrosis and pseudocysts: risk factors and outcome. Pancreas 2018;47(1):92-98. DOI: 10.1097/ MPA.0000000000000965.

90. Grewe M, Tsiotos GG, Luque de-Leon E, et al. Fungal infection in acute necrotizing pancreatitis. J Am Coll Surg 1999;188(4):408-414. DOI: 10.1016/S1072-7515(98)00334-2.

91. Gloor B, Müller CA, Worni M, et al. Pancreatic infection in severe pancreatitis: the role of fungus and multiresistant organisms. Arch Surg 2001;136(5):592-596. DOI: 10.1001/archsurg.136.5.592.
92. De Waele JJ, Vogelaers D, Blot S, et al. Fungal infections in patients with severe acute pancreatitis and the use of prophylactic therapy clinical infectious diseases. Clin Infect Dis 2003;37(2):208-213. DOI: $10.1086 / 375603$.

93. Horibe M, Sanui M, Sasaki M, et al. Impact of antimicrobial prophylaxis for severe acute pancreatitis on the development of invasive candidiasis: a large retrospective multicenter cohort study pancreas. Pancreas 2019;48(4):537-543. DOI: 10.1097/MPA.0000000000001269.

94. Safran DB, Dawson E. The effect of empiric and prophylactic treatment with fluconazole on yeast isolates in a surgical trauma intensive care unit. Arch Surg 1997;132(11):1184-1188. DOI: 10.1001/ archsurg.1997.01430350034006.

95. Eggimann P, Francioli P, Bille J, et al. Fluconazole prophylaxis prevents intra-abdominal candidiasis in high-risk surgical patients. Crit Care Med 1999;27(6):1066-1072. DOI: 10.1097/00003246-19990600000019.

96. Pelz RK, Hendrix CW, Swoboda SM, et al. Double-blind placebocontrolled trial of fluconazole to prevent candidal infections in critically ill surgical patients. Ann Surg 2001;233(4):542-548. DOI: 10.1097/00000658-200104000-00010.

97. Büchler MW, Gloor B, Müller CA, et al. Acute necrotizing pancreatitis: treatment strategy according to the status of infection. Ann Surg 2000;232(5):619-626. DOI: 10.1097/00000658-200011000-00001.

98. Otto W, Komorzycki K, Krawczyk M. Efficacy of antibiotic penetration into pancreatic necrosis. HPB (Oxford) 2006;8(1):43-48. DOI: 10.1080/13651820500467275.

99. Schubert S, Dalhoff A. Activity of moxifloxacin, imipenem, and ertapenem against Escherichia coli, Enterobacter cloacae, Enterococcus faecalis, and Bacteroides fragilis in monocultures and mixed cultures in an in vitro pharmacokinetic/pharmacodynamic model simulating concentrations in the human pancreas. Antimicrob Agents Chemother 2012;56(12):6434-6436. DOI: 10.1128/ AAC.00872-12.

100. Buchler M, Malfertheiner $P$, Friess $H$, et al. Human pancreatic tissue concentration of bactericidal antibiotics. Gastroenterology 1992;103(6):1902-1908. DOI: 10.1016/0016-5085(92)91450-I.

101. Garg PK, Sharma M, Madan K, et al. Primary conservative treatment results in mortality comparable to surgery in patients with infected pancreatic necrosis. Clin Gastroenterol Hepatol 2010;8(12):1089-1094. DOI: 10.1016/j.cgh.2010.04.011.

102. Santvoort HC, Besselink MG, Bakker OJ, et al. A step-up approach or open necrosectomy for necrotizing pancreatitis. N Engl J Med 2010;362(16):1491-1502. DOI: 10.1056/NEJMoa0908821.

103. Mouli VP, Sreenivas V, Garg PK. Efficacy of conservative treatment, without necrosectomy, for infected pancreatic necrosis: a systematic review and meta-analysis. Gastroenterology 2013;144(2):333-340. DOI: 10.1053/j.gastro.2012.10.004.

104. Agarwal S, George J, Padhan RK, et al. Reduction in mortality in severe acute pancreatitis: a time trend analysis over 16 years. Pancreatology 2016;16(2):194-199. DOI: 10.1016/j.pan.2016.01.012.

105. van Santvoort HC, Bakker OJ, Bollen TL, et al. A conservative and minimally invasive approach to necrotizing pancreatitis improves outcome. Gastroenterology 2011;141(4):1254-1263. DOI: 10.1053/j. gastro.2011.06.073.

106. Wolbrink DRJ, KolwijckE, Ten Oever J, et al. Management of infected pancreatic necrosis in the intensive care unit: a narrative review. Clin Microbiol Infect 2020;26(1):18-25. DOI: 10.1016/j.cmi.2019.06.017.

107. van Brunschot S, van Grinsven J, van Santvoort HC, et al. Endoscopic or surgical step-up approach for infected necrotising pancreatitis: a multicentre randomised trial. Lancet 2018;391(10115):51-58. DOI: 10.1016/S0140-6736(17)32404-2.

108. Driedger M, Zyromski NJ, Visser B, et al. Surgical transgastric necrosectomy for necrotizing pancreatitis-a single-stage procedure for walled-off pancreatic necrosis. Ann Surg 2020;271(1):163-168. DOI: 10.1097/SLA.0000000000003048.

109. Diaz Jr JJ, Cullinane DC, Khwaja KA, et al. Eastern Association for the surgery of trauma: management of the open abdomen, part III-review 
of abdominal wall reconstruction. J Trauma Acute Care Surg 2013;75(3):376-386. DOI: 10.1097/TA.0b013e318294bee3.

110. van Baal MC, van Santvoort HC, Bollen TL, et al. Systematic review of percutaneous catheter drainage as primary treatment for necrotizing pancreatitis. Br J Surg 2010;98(1):18-27. DOI: 10.1002/bjs. 7304.

111. van Grinsven J, van Santvoort HC, Boermeester MA, et al. Timing of catheter drainage in infected necrotizing pancreatitis. Nat Rev Gastroenterol Hepatol 2016;13(5):306-312. DOI: 10.1038/ nrgastro.2016.23.

112. van Grinsven J, Timmerman $\mathrm{P}$, van Lienden $\mathrm{KP}$, et al. Proactive versus standard percutaneous catheter drainage for infected necrotizing pancreatitis. Pancreas 2017;46(4):518-523. DOI: 10.1097/ MPA.0000000000000785.

113. Shenvi S, Gupta R, Kang M, et al. Timing of surgical intervention in patients of infected necrotizing pancreatitis not responding to percutaneous catheter drainage. Pancreatology 2016;16(5):778-787. DOI: 10.1016/j.pan.2016.08.006.

114. Horvath KD, Kao LS, Wherry KL, et al. A technique for laparoscopicassisted percutaneous drainage of infected pancreatic necrosis and pancreatic abscess. Surg Endosc 2001;15(10):1221-1225. DOI: 10.1007/ s004640080166.

115. van Brunschot $S$, Fockens $P$, Bakker OJ, et al. Endoscopic transluminal necrosectomy in necrotising pancreatitis: a systematic review. Surg Endosc 2014;28(5):1425-1438. DOI: 10.1007/s00464-013-3382-9.

116. Yi F, Ge L, Zhao J, et al. Meta-analysis: total parenteral nutrition versus total enteral nutrition in predicted severe acute pancreatitis. Intern Med 2012;51(6):523-530. DOI: 10.2169/internalmedicine.51. 6685.

117. Hsieh PH, Su HY, Lin CY, et al. Infection rate among nutritional therapies for acute pancreatitis: a systematic review with network meta-analysis of randomized controlled trials. PLoS One 2019;14(7):e0219151. DOI: 10.1371/journal.pone.0219151.

118. van Minnen LP, Timmerman HM, Lutgendorff F, et al. Modification of intestinal flora with multispecies probiotics reduces bacterial translocation and improves clinical course in a rat model of acute pancreatitis. Surgery 2007;141(4):470-480. DOI: 10.1016/j. surg.2006.10.007.
119. Olah A, Belagyi $T$, Issekutz $A$, et al. Randomized clinical trial of specific lactobacillus and fibre supplement to early enteral nutrition in patients with acute pancreatitis. Br J Surg 2002;89(9):1103-1107. DOI: 10.1046/j.1365-2168.2002.02189.x.

120. Olah A, Belagyi T, Poto L, et al. Synbiotic control of inflammation and infection in severe acute pancreatitis: a prospective, randomized, double blind study. Hepatogastroenterology 2007;54(74):590-594.

121. Karakan T, Ergun M, Dogan I, et al. Comparison of early enteral nutrition in severe acute pancreatitis with prebiotic fiber supplementation versus standard enteral solution: a prospective randomized doubleblind study. World J Gastroenterol 2007;13(19):2733-2737. DOI: 10.3748/wjg.v13.i19.2733.

122. Besselink MG, Van Santvoort HC, Buskens E, et al. Probiotic prophylaxis in predicted severe acute pancreatitis: a randomised, double-blind, placebo-controlled trial. Lancet 2008;371(9613):651659. DOI: 10.1016/S0140-6736(08)60207-X.

123. Qin HL, Zheng JJ, Tong DN, et al. Effect of Lactobacillus plantarum enteral feeding on the gut permeability and septic complications in the patients with acute pancreatitis. Eur J Clin Nutr 2008;62(7): 923-930. DOI: 10.1038/sj.ejcn.1602792.

124. Cui LH, Wang XH, Peng LH, et al. The effects of early enteral nutrition with addition of probiotics on the prognosis of patients suffering from severe acute pancreatitis. Zhonghua Wei Zhong Bing Ji Jiu Yi Xue 2013;25(4):224-228. DOI: 10.3760/cma.j.issn.2095-4352.2013.04.011.

125. Gou S, Yang Z, Liu T, et al. Use of probiotics in the treatment of severe acute pancreatitis: a systematic review and meta-analysis of randomized controlled trials. Crit Care 2014;18(2):R57. DOI: 10.1186/ cc13809.

126. Wu P, Yu Y, Li L, et al. Effect and safety of probiotics combined early enteral nutrition on severe acute pancreatitis patients. Biomed Res 2017;28(3):1403-1407.

127. Bouwense SA, van Baal MC, van Santvoort HC, et al. Prophylaxis and treatment with antibiotics or probiotics in acute pancreatitis. Pancreapedia: Exocrine Pancreas Knowledge Base 2016. DOI: 10.3998/ panc.2016.19.

128. Pan LL, Li J, Shamoon M, et al. Recent advances on nutrition in treatment of acute pancreatitis. Front Immunol 2017;8:762. DOI: 10.3389/fimmu.2017.00762. 